\title{
Dispersion of Localized Mode Polaritons in $\mathrm{K}_{3} \mathrm{Cu}(\mathrm{CN})_{4}$
}

\author{
Wilhelm Nitsch \\ Sektion Physik der Universität München, Lehrstuhl Prof. J. Brandmüller, München, Germany
}

(Z. Naturforsch. 30 a, $537-540$ [1975] ; received February 8, 1975)

\begin{abstract}
The dispersion of polaritons of symmetry $A_{1}$ and $E$ is investigated in the region of four isotopic vibrations originating from $\mathrm{C}^{13}$. and $\mathrm{N}^{15}$-atoms in their natural abundances. The measured dispersion curves are explained in terms of a theory for localized mode polaritons (LMP) developed by Ohtaka. The dispersion relation is generalized for a polyatomic crystal and applied to $\mathrm{K}_{3} \mathrm{Cu}(\mathrm{CN})_{4}$. The comparison of experimental data with calculated dispersion curves shows a quite satisfying agreement for a certain value of the coupling constant which is approximately the same for E-type and $A_{1}$-type polaritons.
\end{abstract}

\section{Introduction}

In most of the theoretical papers on polaritons the crystal lattice is assumed to be perfect and the potential is treated in the harmonic approximation. The agreement found between experimental data and the dispersion curves calculated under these assumptions is quite satisfying in general. However, it is well known that substitutional mass defects in an otherwise perfect crystal can give rise to new optical modes. When the concentration of the defects is small compared to unity, e.g. $1 \%$ or less, the resulting impurity vibrations oscillate independently and their amplitudes are localized around the defects, decaying exponentially with the distance from the impurity site.

If the impurity mode is infrared active in addition a new interesting feature arises. The impurity oscillators may couple by means of the electromagnetic fields associated and move coherently. The coherent motion of the whole defect system shows a wavelike character and consequently LO-TO frequency splittings. In the region of small wavevectors, $k \lesssim 2 \cdot 10^{4} \mathrm{~cm}^{-1}$, these modes can couple to electromagnetic waves. The resulting mixed modes have become known as localized-mode-polaritons in analogy to phonon-polaritons. Thus in the neighbourhood of localized modes the polaritondispersion shows significant deviations from the curves, calculated using only the data of the host crystal.

In $\mathrm{K}_{3} \mathrm{Cu}(\mathrm{CN})_{4}$ there are four isotopic modes originating from $\mathrm{C} \equiv \mathrm{N}$-stretching vibrations from $2030 \mathrm{~cm}^{-1}$ to $2055 \mathrm{~cm}^{-1}$. These modes are due to

Reprint requests to W. Nitsch, Sektion Physik der Universität, Lehrstuhl Prof. Dr. J. Brandmüller, D-8000 München 40 , Schellingstr. $4 / \mathrm{IV}$.
$\mathrm{C}^{13}$ and $\mathrm{N}^{15}$ atoms in their natural abundances of $1.12 \%$ and $0.36 \%$ and have earlier been studied and assigned by Raman- ${ }^{1,2}$ and IR-Spectroscopy ${ }^{3}$.

Though these modes are no localized modes in the sense of the definition given by Maradudin ${ }^{4}$, we can assume, that they are highly localized around the defect site and refer to them as "quasi-localized modes".

It is the special purpose of this work to show the influence of the isotopic modes on the polariton dispersion. The observed dispersion curves are explained in terms of a theory of localized mode polaritons, derived by Ohtaka ${ }^{5}$. Comparison between our Raman data and the calculated curves shows a satisfying agreement.

\section{Theory}

The influence of substitutional mass defects on the polariton dispersion is treated by a modified calculation of the frequency dependent dielectric constant $\varepsilon(\omega)$. A first corresponding theory for diatomic crystals of cubic symmetry was developed by Mills and Maradudin ${ }^{6}$ and Mills and Oitmaa ${ }^{7}$. They derive

$$
\varepsilon(\omega)=\varepsilon_{\infty}+\Omega_{\mathrm{imp}}^{2} /\left(\tilde{\omega}_{\mathrm{T}}^{2}-\omega^{2}\right)
$$

where $\varepsilon_{\infty}$ is the high frequency dielectric constant, $\tilde{\omega}_{\mathrm{T}}$ the transverse local mode frequency and $\Omega_{\text {imp }}$ the plasma frequency of the collective impurity mode. $\Omega_{\text {imp }}$ can be calculated from

$$
\Omega_{\mathrm{imp}}^{2}=4 \pi e^{2} C_{\mathrm{d}} / M^{\prime} V
$$

$e^{*}$ denoting the effective charge of the ion pair, $C_{\mathrm{d}}$ the defect concentration, $M^{\prime}$ the impurity mass and $V$ the volume of the unit cell. 
This result, however, holds if only the impurity atoms are vibrating while the host lattice remains at rest. The dispersion relation for polaritons is given by (see e. g. Ref. $^{8}$ )

$$
c^{2} k^{2} / \omega^{2}=\varepsilon(\omega)
$$

where $k$ is the magnitude of the polariton wavevector. As a consequence the polariton branch approaches the dispersion curve of light outside the resonance region and for great $\omega$.

The theory derived by Ohtaka ${ }^{5}$ leads to the following equation:

$$
\varepsilon(\omega)=\varepsilon_{\infty}+\frac{\Omega_{\mathrm{h}}^{2}}{\omega_{\mathrm{T}}^{2}-\omega^{2}}+\frac{\Omega_{\mathrm{imp}}^{2} \xi(\tilde{\omega})}{\tilde{\omega}_{\mathrm{T}}^{2}-\omega^{2}} .
$$

This is the familiar expression for the dielectric function arising from two IR-active lattice modes. More precisely the first two terms describe the contribution from the host crystal while the last term arises from the isotopic mode. The plasma frequency of the isotopic mode is now referred to as $\Omega_{\mathrm{imp}}^{2} \xi(\omega)$ where

$$
\xi(\omega)=\left|\chi_{\mathrm{imp}}\right|^{2} M^{\prime} \varepsilon^{2} /\left[1-\omega_{\mathrm{T}}{ }^{2} / \tilde{\omega}^{2}\right]^{2}
$$

$\chi_{\text {imp }}$ denotes the amplitude of an impurity atom and

$$
\varepsilon^{2}=\left(1-M_{-}{ }^{\prime} / M_{-}\right)^{2}
$$

where $M_{-}$and $M^{\prime}$ - are the masses of the negative host lattice ion and the substitute, respectively.

The result of Eq. (1) is improved in two ways: the contribution of the optically active mode of the host crystal has been taken into account and the definition of the plasma frequency has been refined. As a consequence the polariton dispersion curve does not approach to $\omega=\left(c k / \sqrt{\varepsilon_{\infty}}\right)$ outside the resonance region but to the dispersion curve of the polariton of the host crystal.

\section{Application to $\mathrm{K}_{3} \mathrm{Cu}(\mathrm{CN})_{4}$}

In a recent paper ${ }^{9}$ we reported the interaction of polaritons of symmetry $A_{1}$ and $E$ with isotopic modes in $\mathrm{K}_{3} \mathrm{Cu}(\mathrm{CN})_{4}$. We observed a resonance splitting of the polariton dispersion curves at the isotopic mode frequencies which was simultaneously accomponied by a drastic change of the mode intensities. Yet we did not succed in getting a satisfying agreement of our experimental data with dispersion curves calculated on the basis of the general polariton theory.
The theory by Ohtaka was developed for diatomic crystals of cubic symmetry. $\mathrm{K}_{3} \mathrm{Cu}(\mathrm{CN})_{4}$ however is a uniaxial crystal with 28 atoms in the unit cell ${ }^{10,11}$ which consequently exhibits a great number of Raman- and IR-active modes ${ }^{1}$. The spectrum is clearly devided into two sections: a low frequency region from 40 to about $300 \mathrm{~cm}^{-1}$ and a second region from 2030 to $2100 \mathrm{~cm}^{-1}$, which contains only three LO-TO pairs originating from $\mathrm{C} \equiv \mathrm{N}$ stretching vibrations of the host crystal, and in addition four isotopic modes. Because the influence of the low frequency modes on $\varepsilon(\omega)$ near the isotopic mode frequencies is small, we can replace it by introducing a representative LO-TO-splitting at $300 \mathrm{~cm}^{-1}$, which is approximately the maximum first order phonon frequency in the low frequency region. Thus we can reduce the total number of modes to three of symmetry $A_{1}$ and two of symmetry $\mathrm{E}$ and in addition the number of isotopic modes of each symmetry.

We have to generalize Eq. (3) for the multi mode case and obtain:

$$
\frac{c^{2} k^{2}}{\omega^{2}}=\varepsilon_{\infty}+\sum_{i=1}^{m} \frac{S_{i} \omega_{T i}^{2}}{\omega_{T i}^{2}-\omega^{2}}+\sum_{j=1}^{n} \frac{S_{j}^{1} \tilde{\omega}_{j}^{2} \xi\left(\tilde{i}_{j}\right)}{(i)_{j}^{2}-\omega^{2}} .
$$

The first two terms in Eq. (6) describe the frequency dependent dielectric constant of a polyatomic crystal (see e.g. Ref. ${ }^{12}$ ) with m IR-active modes. $S_{i}$ denotes the mode-strength of the $i$-th mode:

$$
S_{i}=\frac{\varepsilon_{\infty}\left(\omega_{\mathrm{L} i}^{2}-\omega_{\mathrm{T} i}^{2}\right)}{\omega_{\mathrm{T} i}^{2}} \sum_{\substack{K=1 \\ K \neq i}}^{m} \frac{\omega_{\mathrm{L} K}^{2}-\omega_{\mathrm{T} i}^{2}}{\omega_{T}^{2}-\omega_{\mathrm{T} i}^{2}}
$$

$\omega_{\mathrm{L}}$ and $\omega_{\mathrm{T}}$ are longitudinal and transversal phonon frequencies, respectively. The mode-strengths can be calculated, if the LO-TO splittings of all $\mathrm{m}$ IR-active modes are known. They have been determined for the high frequency modes of $\mathrm{K}_{3} \mathrm{Cu}(\mathrm{CN})_{4}$ by means of directional dispersion measurements. In principle we could also calculate the modestrengths $S_{j}{ }^{1}$ of the isotopic modes, the LO-TO splittings of these modes, however, turn out to be very small and below the resolution of our experimental equipment (see also Table 1).

We could earlier show ${ }^{9}$ that the isotopic modes of symmetry $A_{1}$ are related to the $A_{1}$ (TO)-phonon at $2074 \mathrm{~cm}^{-1}$ and the isotopic modes of symmetry E to the E(TO)-phonon at $2080 \mathrm{~cm}^{-1}$ by a simple Einstein-oscillator-equation, if we only take into 
account the change of reduced mass of a single ${ }^{12} \mathrm{C} \equiv{ }^{14} \mathrm{~N}$-group when one of the atoms is replaced by its isotope. The isotope mode frequencies calculated in this way fit the experimental ones within an error of less than half a percent. This leads us to the conclusion that in a first approximation the local modes can be treated as simple $\mathrm{C} \equiv \mathrm{N}$ stretching vibrations and consequently we can calculate the mode strengths of the isotopic modes from those of the related "unperturbed" modes of the host crystal. We thus obtain:

$$
S_{K}^{1}=S^{\mathrm{h}} \cdot C_{\mathrm{d} K}\left(\mu \omega_{\mathrm{T}}{ }^{2} / \mu_{K}{ }^{*} \tilde{(1)}_{K}{ }^{2}\right)
$$

where $S^{\mathrm{h}}$ is the mode strength of the unperturbed mode, $C_{\mathrm{d} K}$ the concentration of the $K$-th isotope and $\mu$ and $\mu^{*}$ are the effective masses of a pure and a substituted CN-group, respectively.

The polariton scattering experiments were performed in such a way, that the wavevectors were always propagating in the optically isotropic plane. No directional dispersion could therefore affect the dispersion of polaritons.

Applying Eq. (6) to a uniaxial crystal it splits into two dispersion relations: one for extraordinary polaritons which for general wavevector directions are of mixed symmetry $\mathrm{A}_{1}+\mathrm{E}$ and type $\mathrm{TO}+\mathrm{LO}$ and another one for ordinary polaritons of symmetry E(TO) with latitce displacements perpendicular to the optic axis for all wave vector directions.

\section{Results and Discussion}

Figure 1 shows the polariton branch associated with the $\mathrm{E}$ (TO)-phonon at $2080 \mathrm{~cm}^{-1}$ in the region of the isotopic modes of symmetry E. The horizontal lines at 2040 and $2053 \mathrm{~cm}^{-1}$ indicate the frequencies of these modes. The limiting line to the left hand side of the figure is obtained from the wave vector relation $k_{i}=k_{s}+k_{p}$ and determines the maximum frequency shift of polaritons which can be obtained by straight forward scattering. The limiting line has been calculated for $\mathrm{Kr}$-ion-laser excitation at $647.1 \mathrm{~nm}$.

The sets of dispersion curves were calculated with different values of $\left(1 / M^{\prime}\right)\left|\chi_{\text {imp }}\right|^{2}$ in the following steps: (1): $0.0,(2): 0.30,(3): 0.60$, (4) : 1.00. Curve (1) shows the dispersion of the pure host crystal, because there is no contribution from the isotopic modes in Equation (6). The best agreement between our experimental data and the cal-

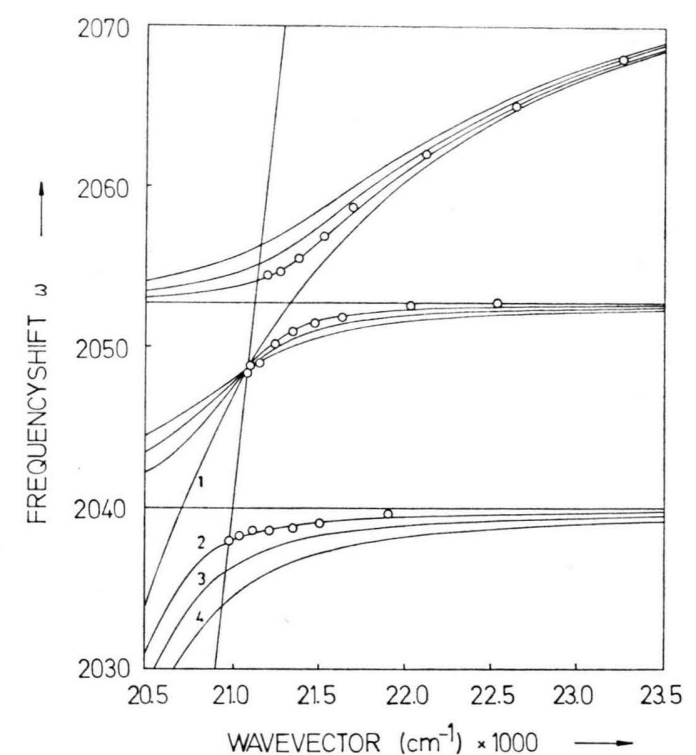

Fig. 1. Dispersion curve and experimental data concerning the E-polariton in $\mathrm{K}_{3} \mathrm{Cu}(\mathrm{CN})_{4}$. The natural abundances of isotopes in the material are $1.12 \% \mathrm{C}^{13}$ and $0.36 \% \mathrm{~N}^{15}$. Curve 1 is the dispersion curve of $\mathrm{K}_{3} \mathrm{Cu}(\mathrm{CN})_{4}$, when the impurity vibrations are neglected. Curve 2 to 4 are plotted for different values of $\left(1 / M^{\prime}\right)\left|\chi_{\text {imp }}\right|^{2}$. (2) : 0.33 , (3) : 0.66 , (4) : 1.00. The following input data have further been used: $\varepsilon_{\infty \perp}=2.35, \omega_{1 \mathrm{~T}}^{\frac{1}{1}}=300 \mathrm{~cm}^{-1}, \omega \frac{1}{1 \mathrm{~L}}=330 \mathrm{~cm}^{-1}, \omega \frac{1}{2 \mathrm{~T}}$ $=2080 \mathrm{~cm}^{-1}, \omega \frac{1}{2 \mathrm{~L}}=2085.2 \mathrm{~cm}^{-1}, \tilde{\omega}_{1}^{\perp}=2040.0 \mathrm{~cm}^{-1}$, $\tilde{a}_{2}^{\perp}=2053.0 \mathrm{~cm}^{-1}$.

culated branches appears for set (2) where $\left(1 / M^{\prime}\right)\left|\chi_{\text {imp }}\right|^{2}=0.30$, whereas (3) and (4) exhibit considerable deviations. The real dispersion curves obviously approach assymptotically to the dispersion curve of the host crystal outside the resonance region. In a sufficient distance from the resonance frequencies the influence of the isotopic modes is negligably small and the polariton dispersion is not affected by the presence of the isotopic modes. On the other hand the polariton dispersion between the resonances is mainly governed by the overlapping contributions from the isotopic modes to $\varepsilon(\omega)$.

Figure 2 shows the dispersion of the polariton associated with the $A_{1}$ (TO) -phonon at $2073 \mathrm{~cm}^{-1}$. As earlier reported ${ }^{9}$ we observed resonance of the polariton with the isotopic modes both of symmetry $A_{1}$ and E. This may be explained by the fact that the crystal symmetry is disturbed at the defect sites. As a result we obtain a total number of four splittings of the host crystal dispersion curve, each appearing at an isotopic mode frequency. In order to achieve a reasonable fit the mode strengths of the 


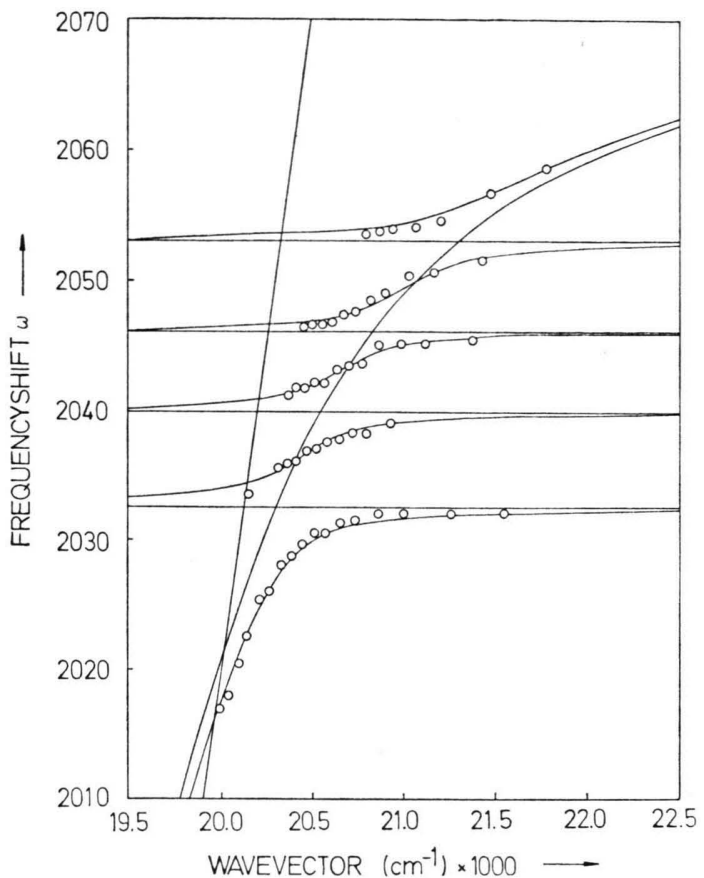

Fig. 2. Dispersion curve and experimental data concerning the $\mathrm{A}_{1}$-polariton in $\mathrm{K}_{3} \mathrm{Cu}(\mathrm{CN})_{4}$ with $1.12 \% \mathrm{C}^{13}$ and $0.36 \%$ $\mathrm{N}^{15}$. Input data: $\varepsilon_{\infty}=2.32, \omega_{1 \mathrm{~T}}^{\|}=300 \mathrm{~cm}^{1}, \omega_{1 \mathrm{~L}}^{\|}=330$ $\mathrm{cm}^{-1}, \omega_{2 \mathrm{~T}}^{\|}=2074 \mathrm{~cm}^{-1}, \omega_{2 \mathrm{~L}}^{\|}=2077.0 \mathrm{~cm}^{-1}, \omega_{3 \mathrm{~T}}^{\|}=$ $2093.5 \mathrm{~cm}^{-1}, \omega{ }_{\mathrm{sL}}=2094.0 \mathrm{~cm}^{-1}, \tilde{\omega}_{\mid 1}=2032.5 \mathrm{~cm}^{-1}$, $\tilde{\omega}_{2}^{\| \prime}=2040.0 \mathrm{~cm}^{-1}$.

E-type isotopic modes had to be reduced by a factor of three. For a better survey we have only plotted those dispersion curves which fit our experimental data best. The best fit was here obtained for $\left(1 / M^{\prime}\right)\left|\chi_{\text {imp }}\right|^{2}=0.33$. Note that the magnitude of the splitting is different for the highest and lowest frequency resonances. This is caused by different impurity concentrations and different mode strengths.

The zeroes of the frequency dependent dielectric function determine the LO-frequencies of the crystal. Consequently we can calculate the LO-TO-splittings of the isotopic modes from Equation (6). The results are shown in Table 1 : The calculated LO-TO-

1 W. Nitsch, H. J. Falge, and R. Claus, Z. Naturforsch. 29 a, 1011 [1974]

2 W. Nitsch, Diploma thesis, Munich 1974.

3 L. H. Jones, J. Chem. Phys. 29, 463 [1958].

4 A. A. Maradudin, Solid State Physics, Supplement 3, Theory of Lattice Dynamics in the Harmonic Approximation. New York, London 1971.

5 K. Ohtaka, Phys. Stat. Sol. (b) 57, 51 [1973].

6 D. L. Mills and A. A. Maradudin, Phys. Rev. B 1, 903 [1970].

7 D. L. Mill and J. Oitmaa, Sol. State Commun. 7, 1143 [1969].
Table 1. LO-TO splittings of the isotope modes in $\mathrm{K}_{3} \mathrm{Cu}(\mathrm{CN})_{4}$, calculated from Equation (6).

\begin{tabular}{lll}
\hline Wave number & Assignment & LO-TO-splitting \\
\hline $2032.5 \mathrm{~cm}^{-1}$ & $\mathrm{~A}_{1},{ }^{13} \mathrm{C} \equiv{ }^{14} \mathrm{~N}$ & $0.0537 \mathrm{~cm}^{-1}$ \\
$2040.0 \mathrm{~cm}^{-1}$ & $\mathrm{E},{ }^{13} \mathrm{C} \equiv{ }^{14} \mathrm{~N}$ & $0.0699 \mathrm{~cm}^{-1}$ \\
$2046.0 \mathrm{~cm}^{-1}$ & $\mathrm{~A}_{1},{ }^{12} \mathrm{C} \equiv{ }^{15} \mathrm{~N}$ & $0.0293 \mathrm{~cm}^{-1}$ \\
$2053.0 \mathrm{~cm}^{-1}$ & $\mathrm{E},{ }^{12} \mathrm{C} \equiv{ }^{15} \mathrm{~N}$ & $0.0325 \mathrm{~cm}^{-1}$ \\
\hline
\end{tabular}

splittings are at least one order of magnitude smaller than the resolution of our experimental equipment and there seems to be hardly a chance to observe them in Raman scattering experiments, even if crystals with higher defect concentrations are used.

If, on the other hand, we could observe the LOTO-splittings of the isotopic modes experimentally, the polariton dispersion is completely determined by 13

$$
c^{2} k^{2} / \omega^{2}=\varepsilon_{\infty} \prod_{i}^{m+n}\left[\omega_{\mathrm{L} i}^{2}-\omega^{2}(k)\right] / \prod_{i}^{m+n}\left[\omega_{\mathrm{T} i}^{2}-\omega^{2}(k)\right]
$$

where $m$ denotes the number of host crystal modes and $n$ the number of isotopic modes.

At the present time work on crystals with different ${ }^{13} \mathrm{C}$ concentrations is going on, which will provide further information on the polariton dispersion and the mode intensities and their dependence on $\mathrm{C}_{\mathrm{d}}$.

\section{Acknowledgement}

I want to thank J. Brandmüller for his interest in this work and R. Claus and F. X. Winter for stimulating discussion and a critical reading of the manuscript. I am grateful to the Bayer. Akademie der Wisserschaften for the grant of computer-time and to the Studienstiftung des Deutschen Volkes for financial support. The laser was provided by the Deutsche Forschungsgemeinschaft.

8 M. Born and K. Huang, Dynamical Theory of Crystal Lattices, Clarendon Press, Oxford 1966.

9 W. Nitsch and R. Claus, Z. Naturforsch. 29a, 1017 [1974].

10 S. Haussühl, Z. Kristallogr. 125, 1 [1967].

11 R. B. Roof, A. C. Larson, and D. T. Cromer, Acta Crystall B 24, 269 [1968].

12 R. Loudon, Light Scattering Spectra of Solids, ed. by G. B. Wright, Springer-Verlag, New York 1969.

13 R. Claus, Festkörperprobleme XII, Advances in Solid State Physics, ed. by O. Madelung, Pergamon/Vieweg, London-Braunschweig 1972, p. 381. 